\title{
A Markov Jump Process Model for Urban Vehicular Mobility: Modeling and Applications
}

\author{
Yong Li, Member, IEEE, Depeng Jin, Member, IEEE, Zhaocheng Wang, Senior Member, IEEE, \\ Pan Hui, Member, IEEE, Lieguang Zeng, and Sheng Chen, Fellow, IEEE
}

\begin{abstract}
Vehicular networks have been attracting increasing attention recently from both the industry and research communities. One of the challenges in this area is understanding vehicular mobility, which is vital for developing accurate and realistic mobility models to aid the vehicular communication and network design and evaluation. Most of the existing works mainly focus on designing microscopic level models that describe the individual mobility behaviors. In this paper, we explore the use of Markov jump process to model the macroscopic level vehicular mobility. Our proposed simple model can accurately describe the vehicular mobility and, moreover, it can predict various measures of network-level performance, such as the vehicular distribution, and vehicular-level performance, such as average sojourn time in each area and the number of sojourned areas in the networks. Model validation based on two large scale urban city vehicular motion traces confirms that this simple model can accurately predict a number of system metrics crucial for vehicular network performance evaluation. Furthermore, we propose two applications to illustrate that the proposed model is effective in analysis of system-level performance and dimensioning for vehicular networks.
\end{abstract}

Index Terms - Vehicular networks, mobility modeling, Markov jump process, performance evaluation

\section{INTRODUCTION}

$\mathrm{R}$ ECENTLY, as more and more vehicles are equipped with devices to provide wireless communication capacities, interests on vehicular communications and networks have grown significantly [1]. Newly emerged vehicular communication networks are seen as a key technology for improving road safety and building intelligent transportation system (ITS) [2]. Many applications of vehicular networks are also emerging, including automatic collision warning, remote vehicle diagnostics, emergency management and assistance for safely driving, vehicle tracking, automobile high speed Internet access, and multimedia content sharing. In USA, Federal Communications Commission has allocated $75 \mathrm{MHz}$ of spectrum for dedicated short-range communications in vehicular networks [3], and IEEE is also working on the related standard specifications. Many consortia and standardisation bodies are actively develop technologies and protocols for information transmission between vehicles and roadside

- Y. Li, D. Jin, Z. Wang, and L. Zeng are with the State Key Laboratory on Microwave and Digital Communications, Tsinghua National Laboratory for Information Science and Technology, Department of Electronic Engineering, Tsinghua University, Beijing 100084, China.

E-mail: \{liyong07, jindp, zcwang, zenglg\}@tsinghua.edu.cn.

- P. Hui is with the Department of Computer Science and Engineering, Hong Kong University of Science and Technology, Hong Kong, and is also with Telekom Innovation Laboratories, Germany, and Aalto University, Finland.E-mail:panhui@cse.ust.hk.

- S. Chen is with Electronics and Computer Science, University of Southampton, Southampton SO17 1BJ, U.K., and also with King Abdulaziz University, Jeddah 21589, Saudi Arabia.

E-mail:sqc@ecs.soton.ac.uk.

Manuscript received 19 Dec. 2012; revised 4 Nov. 2013; accepted 14 Nov. 2013. Date of publication 11 Dec. 2013; date of current version 22 July 2014. For information on obtaining reprints of this article, please send e-mail to: reprints@ieee.org, and reference the Digital Object Identifier below.

Digital Object Identifier 10.1109/TMC.2013.159 unit (RSU) infrastructure equipments, known as vehicles to infrastructures (V2I), and between vehicles, known as vehicles to vehicles (V2V).

Urban vehicular ad hoc networks (VANETs) [4] are recognised as an important component of the next generation ITS to alleviate serious traffic problems, such as traffic jams and accidents, as well as to enable new mobile applications to the public [1]. Since urban VANETs are highly mobile, it is hard to maintain a connected and stable network to communicate. Thus, they are usually distributed, self-organized by the mobile vehicles, and characterized by very high movement and limited communication opportunities in nodes mobility patterns. This brings a strong interaction between the vehicular mobility and network protocol design, which is the main focus for the current development of VANETs [4]. Firstly, mobility in the macroscopic scale, which characterises the flows of vehicular traffic directed from one regions to another, influences the spatial distribution of vehicles, which in turn alters the data traffic. Thus, a specific relationship between the mobility and wireless communication exists in VANETs. Secondly, mobility in the microscope level, which specifies the individual vehicular mobility, influences the positions of individual vehicles. Hence, the communication rate changes when a vehicle communicates with RSUs via V2I [5] or other vehicles via $\mathrm{V} 2 \mathrm{~V}[6]$.

In terms of the design of VANETs, since VANET technologies adopted have a huge impact on the automotive market, a growing effort has been made in the development of communication protocols and mobility models by explicitly taking into account the influence of mobility on communication that is specific to vehicular networks, so that the relationship between node mobility and communication can be utilized efficiently. In terms of the 
performance evaluation of protocols and vehicular network systems, economic issues and technology limitations make theoretical analysis and simulation the main choices in the validation of VANETs. In practice, theoretical analysis and simulation are also widely adopted as the first step in the development of real world technologies [6]. A critical aspect in theoretical analysis and simulation of VANETs is the need for a realistic mobility model reflecting the real behaviors of vehicles, in terms of both large-scale vehicular traffic and microscope level of individual mobility. It can be seen that realistic and accurate mobility models are crucial to the development of vehicular networks and related works in this area form an important part of the on-going development of vehicular networks [6].

After many years of exciting research, a large variety of mobility models are available, which can be classified in three different classes, synthetic models that are obtained by mathematical modeling, survey models extracting mobility patterns from surveys, and trace-based models generating mobility patterns from real mobility trace [6]. Practicality of these mobility models varies from very trivial to most realistic one, and they include freely available models as well as commercial vehicular simulators. However, these models consider each vehicular node as a distinct entity, and they belong to the microscopic-level modeling [7]. Although microscopic-level models can describe individual mobility behavior precisely, they do not have the capacity to describe the overall mobility of the whole network. By contrast, a macroscopic-level description can characterize the gross quantities or metrics, such as vehicular distribution and density, and mean velocity, by treating the vehicular traffic according to fluid dynamics and, therefore, can reveal the large-scale overall vehicular behaviors and traffic. Furthermore, macroscopic-level models are indispensable for network dimensioning, answering "what if" questions like how the network performance changes or the deployed network evolves as the number of vehicles or communication demands scale up [8]. Thus, macroscopic-level vehicular mobility models are crucial for the development of vehicular networking protocols and algorithms.

Against this background, in this paper, we consider the problem of modeling the macroscopic-level vehicular mobility. Specifically, we explore the use of Markov jump process to model the vehicular mobility among the areas divided by the intersections of city roads. In this model, vehicles arrive in the system according to a random process, move from one area to another area by making independent probabilistic transitions, and finally depart the system. The fundamental question we addressed is whether this simple model can accurately describe the vehicular mobility, and predict various measures of network-level performance, such as vehicular distribution, as well as vehicular-level performance, such as average sojourn time in each area and the number of sojourned areas in the vehicular network. Our novel contributions are summarized as follows.

1) We model the macroscopic-level vehicular mobility as a Markov jump process. Under this model, we obtain three important metrics related to vehicular mobility and system performance, which are vehicular area distribution, average sojourn time in each area, and average mobility length.

2) Using two large-scale urban city vehicular motion traces, we validate the accuracy of the proposed vehicular mobility model by comparing the model predicted results with those observed in the traces. Our results confirm that this simple macroscopiclevel model can accurately predict a number of key system metrics which are crucial in evaluating vehicular network performance.

3) Under the proposed Markov jump process model for vehicular mobility, we introduce two specific applications, the first one determining how much the capacity that the RSUs should provide with the increase of communication demands resulting from the increase of vehicles, and the second one investigating the performance of the combinedcommunication network of V2I and V2V. These two applications demonstrate that the proposed model is effective in analysis of system-level performance and dimensioning for vehicular networks.

The rest of this contribution is organized as follows. After introducing the related work in Section 2, we offer our motivation and describe our proposed system model in Section 3. Section 4 derives the related system performance metrics based on the proposed model. In Section 5, we present the model simulation based on real vehicular mobility traces and provide the model validation results. This is followed by two specific applications of vehicular network performance analysis in Section 6. We conclude the paper in Section 7.

\section{Related Work}

For the mobility models, generally they can be classified by three categories of macroscopic, mesoscopic, and microscopic models. For the microscopic level mobility model, a large number of models are available. In this category, synthetic models such as random walk [14] and random waypoint [15]-[17] and survey-based models [18], [19] are often unable to provide modeling of motion patterns even though they can be very complex. Among them, some of the well-known mobility models like random waypoint fail to capture the steady state and realistic mobility behaviors [15]. Consequently, the trace-based approach attempts to extract mobility models from real mobility traces by approximating the movements based on the observed movement patterns [23], [24], [26]. For the mesoscopic level mobility model, Banerjee et al. [25] model the vehicle transitions between different areas in a small part of the city. In this category, there are also some models from the area of civil engineering [20]-[22] targeting at predicting the traffic [21], providing safety-driven applications [20], and simulating the transportation systems [22]. All these mesoscopic and microscopic-level individual-mobility modeling approaches have the limitation that they are unable to obtain global mobility patterns. Also these models are often too complex to be concisely described by mathematical equations. Instead of modeling the individual mobility, our work focuses on the macroscopic-level mobility modeling. 
To our best knowledge, this is the first work that gives a simple mobility model with large-scale urban vehicular mobility empirical data validation.

Recent work [27] focuses on studying the metric of intercontact time, which denotes the time between two successive communication contacts of two vehicles, and it finds that the inter-contact time exhibits the exponential distribution over a large range of timescales. Poisson distributed contact rate has been validated to fit well to real vehicular traces and is widely used to model opportunistic vehicular systems [26], [28], [29]. Instead of studying inter-contact time, Li et al. [30] study another key metric known as contact duration, which is how long a contact lasts. In contrast to these works which reveal the vehicular contact patterns that indirectly reflect the macroscopic mobility, we directly model the macroscopic-level vehicular mobility, and study the vehicular mobility flow and spatial distribution among macroscopic areas.

Previous investigation on modeling and performance analysis with Markov process or queueing network model mostly focuses on access networks or applications in peerto-peer live streaming systems [31]-[33]. The most closely related works are in theoretical analysis for cellular and WiFi networks [8]-[13], [34], [35]. In cellular network research, Markov mobility models have been used to analyze the performance around for decades from [9] to recent works of [12], [13]. In these works, the Markov model is used to predict user mobility in the cellular networks with the aims of resource reservation [10], [12], spectrum allocation [11] and performance evaluation [13]. For specific examples, Ashtiani et al. [34] use a closed queueing network with a fixed number of nodes to model the users and traffic in the cellular network, Kim et al. [35] utilise a M/M/c/c queues to mode cellular network mobile users, while Chen et al. [8] propose a mixed queueing network model to describe the user mobility among access points in a campus wireless network environment. All these models for wireless networks are proposed under various assumptions to facilitate mathematical derivations. By contrast, our work focuses on modeling the large-scale urban vehicular mobility. Rather than giving complex mathematical derivation, we justify that using the simplest Markov jump process model can capture the essential properties for vehicular mobility by the validation using two empirical real mobility traces. Moreover, we introduce two typical applications, which demonstrate the usefulness of our proposed model in vehicular network performance analysis and design.

\section{Proposed Model}

Since we use two large-scale urban city vehicular motion traces, Shanghai and Beijing traces, to validate our model, and these two data sets play a crucial role in motivating our work, we first provide a brief description of these two traces.

\subsection{Data Sets and Preprocessing}

Shanghai trace [27] was collected by SG project [36], in which 4,441 operational taxis covered the month of February 2007 in Shanghai city. In this trace, a taxi sends its position report by GPRS to the central database every 1 minute when it has passengers onboard but every 15 seconds when it is vacant for the reason of real-time scheduling. However, the different intervals of reporting may distort the records of the physical movements of the taxis, since most of taxis are not vacant most of the time. Another potential drawback of this trace is that the number of taxis is limited. Indeed, about 4400 taxis and 1 minute duration may not be sufficient to record the statistical features of mobility in a high-speed large urban environment.

In collecting Beijing trace, we used the mobility track logs obtained from 28,590 participating Beijing taxis carrying GPS receivers during May 2010. The reason for us to also choose taxis as vehicular devices is that taxis are more sensitive to urban environments in terms of underlying road topology, traffic control and urban planning, and they have broader coverage in terms of space and operation time than buses and private cars. Specifically, we utilized the GPS devices to collect the taxis locations and timestamps and GPRS modules to report the records every 15 seconds for moving taxis. The specific information contained in such a report includes: the taxi's ID, the longitude and latitude coordinates of the taxi's location, timestamps, instant speed and heading. Beijing trace is the largest vehicular data trace available.

By collecting the GPS information of longitude and latitude coordinates, we obtain the taxis' moving traces that indicate the taxis' locations varying with the time. Since these locations are measured by the GPS devices, the noise may exist in the collected data due to the inaccuracy of GPS device. Furthermore, the taxis may not all report their location times at the same time slots with the same fixed frequency, as in the case of Shanghai trace. Therefore, we need to process the data trace to obtain the accurate locations of all the taxis in the same time slots and with the same frequency. In order to achieve these goals, we first use the city maps of Shanghai and Beijing for the respective traces to correct the taxis' locations so that they are in the regions of related city roads. Then, we use the method of linear interpolation (LI) to insert location points so that all the taxis have location information at every 15 -second interval. To illustrate how this LI method works, consider that we have the location information of one taxi in the original trace with the locations $l_{1}, l_{2}, \ldots, l_{n}$ recorded at the time points $t_{1}<t_{2}<\cdots<t_{n}$, and we want to insert the location information $l_{t}$ at the time point $t$ which is calculated according to the 15-second frequency. All we need to do are finding $t_{m}$ that satisfies $t_{m} \leq t<t_{m+1}$, and then estimating the location $l_{t}$ by the following LI

$$
l_{t}=\frac{t_{m+1}-t}{t_{m+1}-t_{m}} \cdot l_{m}+\frac{t-t_{m}}{t_{m+1}-t_{m}} \cdot l_{m+1} .
$$

In order to verify that the above data preprocessing approach does not include artificial and inaccurate information into the original data trace, we use the data obtained by this preprocessing method for the one-day taxis' locations to plot the trajectories of all the taxis, which are shown in Fig. 1(a) and (b) for Beijing and Shanghai data, respectively. It can be seen from Fig. 1 that the data sets are sufficiently large, and even using one-day data can recover the whole city maps. In order to further demonstrate the accuracy of our data preprocessing, we compare the recovered maps of 


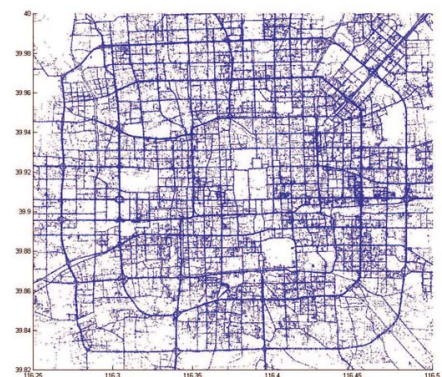

(a)

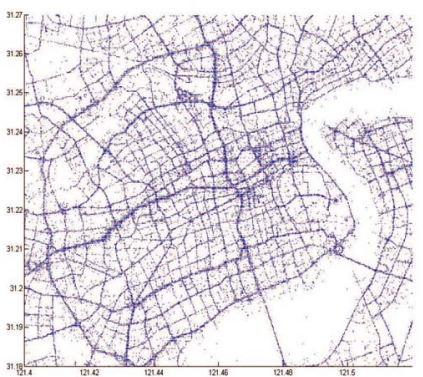

(b)
Fig. 1. City maps recovered from one-data taxis mobility traces of (a) Beijing, and (b) Shanghai.

Beijing and Shanghai depicted in Fig. 1 with the true Beijing and Shanghai maps. It can be verified that all the taxis' trajectories determined by the preprocessing are in the related city roads, and the two city maps drawn by these one-day trajectories are very similar to the corresponding true city maps.

\subsection{Modeling Motivation}

Consider a vehicle moving in the roads of a city. It will travel along a road and come across an intersection. It may wait at the traffic light for some time and choose the direction at the intersection, and then travels to another road to drive on. In the downtown of a big city, the roads are usually very crowded, and the intersections are very dense, which lead to very long waiting time at intersections and relatively short deriving time along roads. Therefore, intersection is an important factor in modeling the urban vehicular mobility. Thus, viewing from the sky above the city, we observe that a crowd of vehicles waiting at the area of each intersection, and streams of traffic moving from one area to another area. In other words, in order to describe the vehicular distribution, we need to pay particular attention to the areas around intersections and to understand the vehicular behaviors of transition from one area to another from the system viewpoint.

Thus, if we divide the whole urban city into different areas each including at least one intersection, we can model the vehicles moving from one area to another adjacent area and therefore model the vehicular traffic transiting from one area to another. Take Fig. 2 as an example, which is the map of the central area in Shanghai. We first mark the important intersections surrounded by large numbers of vehicles, which are indicated by the red points in Fig. 2, and then divide the whole map into the areas around the selected intersections. The method for this area partition can be chosen flexibly according to the needs of specific applications. For example, if we want to obtain the model to be used in the vehicular network design, e.g. for the use to decide how to deploy the RSU system, then the Voronoi diagram [37] can used, where the boundary between two neighbouring areas passes the middle point of the line connecting the two selected neighbouring intersections and is perpendicular to this line.

Let us consider the 2-dimensional vehicular mobility defined by a sequence of steps that a vehicle travels in the city, which is modelled or partitioned as the areas around

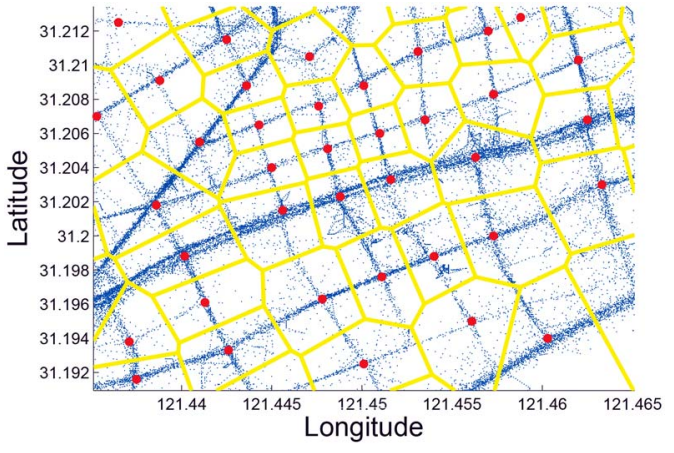

Fig. 2. Illustration of area partition for the central part of Shanghai city.

the intersections as described previously. A step is defined by a tuple $\left(t^{1}, t^{2}, A\right)$ during which a vehicle travels in an area, where $A$ denotes an area, $t^{1}$ is the time that the vehicle enters area $A$, and $t^{2}$ is the time that it departs the area. In the first step, the vehicle enters the city or the modelled region by entering an area, and after some steps, it moves out of the modelled region. Every vehicle moves in this way by transiting from one area to another area, which depicts each vehicle's mobility. Thus, we can describe the traffic flows of the whole system by combining all the vehicles' mobility patterns and all the intersections together as a system. We are now ready to introduce our Markov jump process to model the above macroscopic-level vehicle mobility scenario.

\subsection{Model Description}

Using the method of area partitioning described in the previous subsection, the whole vehicular system can be partitioned into areas, the number of which is denoted by $N$. Vehicles move into the system, transit from one area to another, and finally move out the system. We use a Markov jump process to model this system, which is illustrated in Fig. 3. The Markov jump process includes $N$ states to represent the $N$ partitioned areas in the system. The states are numbered by the set $\mathcal{N}=\{1,2, \ldots, N\}$, and the corresponding areas are denoted by the set $\mathcal{A}=\left\{A_{1}, A_{2}, \ldots, A_{N}\right\}$, which is also used to represent the set of all the $N$ states. A vehicle entering the system and moving from one area to another is modelled by the entrance into the Markov jump process and the transition from one state to another. We now specify the parameters of this Markov jump process that describe the dynamic behaviors of this vehicular mobility system.

From the viewpoint of the Markov jump process, vehicles enter the system with certain rate, stay in a state for some time, and then transfer to other state with the events

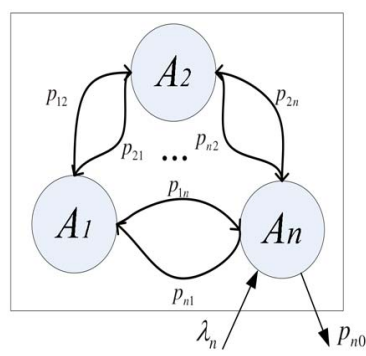

Fig. 3. Vehicular mobility model. 
TABLE 1

Parameters of the Markov Jump Process Model

\begin{tabular}{c|c}
\hline Notations & Meaning \\
\hline $\mathcal{A}$ & The set of areas or the set of states \\
$\lambda_{n}$ & The number of states or areas in the system \\
$\gamma_{n}$ & The exogenous arrival rate for state $n$ \\
$1 / \mu_{n}$ & The effective arrival rate for state $n$ \\
$\rho_{n}=\gamma_{n} / \mu_{n}$ & The expected vehicle residence time at area $n$ \\
$p_{n m}(m \neq n)$ & The load of area or state $n$ \\
$p_{n 0}$ & Probability of vehicles moving from area $n$ to area $m$ \\
$M_{n}$ & Probability of vehicles leaving the system from area $n$ \\
$W_{n}$ & The number of vehicles in the system \\
\hline
\end{tabular}

of turn on and turn off. In this vehicular mobility system with multi-areas, the vehicles' dynamic behaviors occur on two different time scales. Vehicles may enter and depart the system, or appear and disappear from the system due to turn on/off, which happen in a long time scale. Vehicles may change areas, that is, switch from one state to another, which occur at a short time scale. To model the dynamics of long time scale, we assume that vehicles arrive at state $n, n \in \mathcal{N}$, with rates of $\lambda_{n}$, which includes the rates that the vehicles move into the modeled area and the vehicles turn on, and define it as exogenous arrival rate. When a vehicle moves into area $A_{n}$, it will stay in this area for a period of time. We assume that the average amount of time that vehicles stay with state $A_{n}$ is $\mu_{n}$. The distribution of this staying time may be arbitrary. To characterize the dynamics of short time scale, we assume that after the vehicle staying in area $A_{n}$ for a random period of time, it switches to area $A_{m}$, where $m \neq n$, with probability $p_{n m}$, or it leaves the region with probability $p_{n 0} . p_{n 0}$ is defined as the probability of vehicles leaving the system from area $n$ since the two reasons of moving into other areas out of our model and vehicles turning off in area $n$. To complete the description of the vehicular mobility system, we denote the total number of vehicles in the system as $M$, and the number of vehicles in area $A_{n}$ by $W_{n}$.

The key parameters in our model are summarized in Table 1 . To recap, we model the vehicular mobility system partitioned into $N$ areas as a Markov jump process of $N$ states. In this open system with $M$ vehicles, vehicles join and leave the system freely. The exogenous arrival rate for state $A_{n}$ is $\lambda_{n}$, and the effective arrival rate for state $A_{n}$ is $\gamma_{n}$ which takes into account the exogenous arrival rate $\lambda_{n}$ and all the transitions from other areas $A_{m}$ for $m \neq n$. After staying in the state $A_{n}$ for the time period of $1 / \mu_{n}$, a vehicle switches to another state $A_{m}$ with probability $p_{n m}$, or it may leave the process with probability $p_{n 0}$. We will denote the load of state $A_{n}$ as $\rho_{n}=\gamma_{n} / \mu_{n}$.

For the above Markov jump process used to model the vehicular mobility system, if the exogenous arrival to each state follows a Poisson process, it becomes a Markov jump process with superimposed Poisson arrival [9], [38]. This process has some well-known results regarding the user distribution and waiting time, which we can then directly apply to our vehicular mobility system. By using Beijing and Shanghai traces, we find that the actual exogenous arrival process of the vehicular mobility matches well with the exponential distribution, as will be demonstrated by the model validation results given in Section 5. Thus, the vehicular mobility system can be modelled as a Markov jump process with superimposed Poisson arrival, based on which we can derive some key metrics to depict the system's achievable performance.

\section{Performance Metric Derivation}

Based on the Markov jump process model for vehicular mobility, we derive three important metrics for evaluating the system performance, which are vehicular area distribution, average sojourn time, and average mobility length, where the vehicular area distribution reflects the important macroscopic-level transitions and the sojourn time and mobility length are vital to the problems of traffic congestion and driving route selection. The definitions of these three metrics are formally given as follows.

Definition 1. The vehicular area distribution is the steady-state probability distribution that defines the population distribution of the vehicles among the areas of the system.

More specifically, let $W_{n}$ be the random variable denoting the number of vehicles in area $A_{n}$, where $1 \leq n \leq N$. Then, the marginal probability or single area vehicular distribution, denoted as $P\left(W_{n}=w_{n}\right)$, is the probability that there are $w_{n}$ vehicles in area $A_{n}$. Obviously, $w_{n} \in\{0,1,2, \ldots, M\}$.

Furthermore, the joint area vehicular distribution, denoted by $\pi(w)$, is the joint probability of the vehicle population distribution among all the areas, which is expressed as

$$
\pi(\boldsymbol{w})=P\left(W_{1}=w_{1}, W_{2}=w_{2}, \ldots, W_{N}=w_{N}\right),
$$

where $\boldsymbol{w}=\left[w_{1} w_{2} \cdots, w_{N}\right]$.

Definition 2. The sojourn time of a vehicle is defined as the time period between that the vehicle enters into the system by one of the areas and that it finally leaves the system. This parameter is related to the session time that a vehicle spends when visiting the system. By averaging all the sojourn times over all the vehicles of the system in the steady state, the average sojourn time is obtained.

Definition 3. The average mobility length is defined as the average number of areas that a vehicle travels through during the sojourn time that it is in the system. This parameter is related to the average number of transitions that a vehicle makes during a session or stay with the system.

\subsection{Vehicular Area Distribution}

In the system, area $A_{n}$ has the exogenous arrival rate $\lambda_{n}$. The vehicles switch from one area to another according to the $N \times N$ switching probability matrix given by

$$
\boldsymbol{P}=\left[\begin{array}{cccc}
0 & p_{12} & \cdots & p_{1 N} \\
p_{21} & 0 & \ddots & \vdots \\
\vdots & \ddots & \ddots & p_{N-1 N} \\
p_{N 1} & \cdots & p_{N N-1} & 0
\end{array}\right] .
$$

Note that in the above defined switching probability matrix $\boldsymbol{P}, \forall n, 1 \leq n \leq N, \sum_{i=1}^{N} p_{n i}<1$ since the vehicles may move out of the modeled areas with the probability $p_{n 0}$. Thus, it 


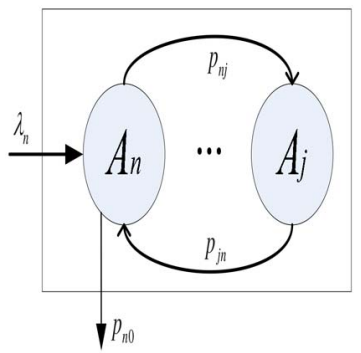

Fig. 4. Effective arrival rate $A_{n}$.

is not the Markov chain's transition matrix. We treat $\mathcal{A}$ as a Markov jump process of $N$ states, and view each vehicle as a customer which sojourns at state $A_{n}$ for a random period of time with mean $1 / \mu_{n}$, which is the staying time in node $A_{n}$. We first determine the effective arrival rate $\gamma_{n}$ for $A_{n}$. As illustrated in Fig. 4, by taking into account the exogenous arrival rate $\lambda_{n}$ and all the transitions from other areas $A_{j}$ for $j \neq n$, the effective arrival rate $\gamma_{n}$ of area $A_{n}$ is given by

$$
\gamma_{n}=\lambda_{n}+\sum_{\substack{j \in \mathcal{N} \\ j \neq n}} \gamma_{j} p_{j n}, 1 \leq n \leq N
$$

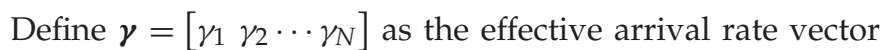
for all the areas in $\mathcal{A}$, and the exogenous arrival rate vector of the system as $\lambda=\left[\lambda_{1} \lambda_{2} \cdots \lambda_{n}\right]$. We can express the relationship (3) in matrix form as follows

$$
\gamma=\lambda+\gamma P \text {. }
$$

According to [39], $\boldsymbol{I}-\boldsymbol{P}$ is invertible, where $\boldsymbol{I}$ denotes the $N \times N$ identity matrix. Thus, the effective arrival rate vector can be solved from (4) as $\gamma=\lambda(I-P)^{-1}$.

The load of area $A_{n}$ is defined as $\rho_{n}=\gamma_{n} / \mu_{n}$. We have the following expressions for the single area vehicular distribution and joint area vehicular distribution related to the loads $\rho_{n}, 1 \leq n \leq N$, of the system.

Lemma 1. The single area vehicular distribution of area $A_{n}$ is given by

$$
P\left(W_{n}=w_{n}\right)=\frac{\rho_{n}{ }^{w_{n}} e^{-\rho_{n}}}{w_{n} !},
$$

and the joint area vehicular distribution of the system is given by

$$
\pi(\boldsymbol{w})=\prod_{j=1}^{N} \frac{\rho_{j}^{w_{j}} e^{-\rho_{j}}}{w_{j} !} .
$$

Furthermore, the average or expected number of vehicles that stay in area $A_{n}$ is $\rho_{n}$.

Proof. For each area $A_{n}$, where $1 \leq n \leq N$, the user arrival rate is $\gamma_{n}$, the staying time is $1 / \mu_{n}$, and the load of $A_{n}$ is $\rho_{n}=\gamma_{n} / \mu_{n}$. Each area of the system is viewed as a state. Therefore, according to the well-known results from Markov jump process [38], the joint probability distribution of the vehicular mobility system is given by

$$
\pi(\boldsymbol{w})=P\left(W_{1}=w_{1}, \ldots, W_{N}=w_{N}\right)=\prod_{j=1}^{N} \frac{\rho_{j} w_{j} e^{-\rho_{j}}}{w_{j} !} .
$$

The marginal distribution of individual area follows immediately as

$$
P\left(W_{n}=w_{n}\right)=\frac{\rho_{n}{ }^{w_{n}} e^{-\rho_{n}}}{w_{n} !} .
$$

We note that the distribution of vehicles at state $A_{n}$ follows the Poisson distribution with mean $\rho_{n}$. Therefore, the expected number of vehicles that stay in area $A_{n}$ is $\rho_{n}$. This completes the proof.

\subsection{Average Sojourn Time}

Using the Markov jump process for the vehicular mobility, we can further analyze the average sojourn time, which is a key system metric related to the mobility behaviors and properties. First, recall that the system state set of the Markov jump process is $\mathcal{A}=\left\{A_{1}, A_{2}, \ldots, A_{N}\right\}$. Vehicles transfer from one state to another are governed by the switching probability matrix $\boldsymbol{P}$ defined in (2). Note that we have already defined $p_{n 0}$ as the probability that a vehicle leaves the modelled region from state $A_{n}$. In order to specify the complete system, we may add the state $A_{0}$ that states vehicles are outside the system, namely, outside the modelled region. Then the completed system state set is $\overline{\mathcal{A}}=\mathcal{A} \cup A_{0}$. Accordingly, we define $p_{0 n}$ as the probability that vehicles move into the system through area $A_{n}$. It can be seen that $p_{0 n}$ is the switching probability from state $A_{0}$ to state $A_{n}$. From the definitions of $p_{n 0}$ and $p_{0 n}$, we have the following expressions for them

$$
\begin{aligned}
& p_{n 0}=1-\sum_{\substack{m \in \mathcal{N} \\
m \neq n}} p_{n m}, 1 \leq n \leq N, \\
& p_{0 n}=\lambda_{n} / \sum_{m=1}^{N} \lambda_{m}, 1 \leq n \leq N .
\end{aligned}
$$

Let the vehicular sojourn time in the system be denoted by $S$, and the average vehicular sojourn time be denoted by $E[S]$, where $E[\bullet]$ denotes the expectation operator. For convenience of derivation, we define $T_{n}$ as the conditional vehicular staying duration in the system, namely, the conditional vehicular sojourn time in the system, conditioned on a vehicle starting from area $A_{n}$, and denote the average value of $T_{n}$ as $E\left[T_{n}\right]$. Using the above definitions and noting that the switching probability from $A_{0}$ to $A_{n}$ is $p_{0 n}$, the average sojourn time can be expressed as

$$
E[S]=\sum_{n \in \mathcal{N}} p_{0 n} E\left[T_{n}\right] .
$$

We have the following theorem regarding the feasibility of computing $E[S]$ using (9).

Theorem 1. Given the expected vehicle residence times $\frac{1}{\mu_{n}}$ at areas $A_{n}, n \in \mathcal{N}$, and the switching probability matrix $\boldsymbol{P}$ defined in (2), all the average conditional vehicular sojourn times $E\left[T_{n}\right]$ for $1 \leq n \leq N$ are completely determined. Therefore, the average sojourn time $E[S]$ can be computed using the expression (9).

Proof. Consider the average conditional vehicular staying duration in the system starting from area $A_{n}$, namely, $E\left[T_{n}\right]$. Using the well-known results of the Markov jump 
process [38], we have

$$
E\left[T_{n}\right]=\frac{1}{\mu_{n}}+\sum_{\substack{m \in \mathcal{N} \\ m \neq n}} p_{n m} E\left[T_{m}\right], 1 \leq n \leq N .
$$

By introducing the vectors $u=\left[\begin{array}{lll}\frac{1}{\mu_{1}} & \frac{1}{\mu_{2}} \cdots \frac{1}{\mu_{N}}\end{array}\right]$ and $\boldsymbol{t}_{E}=\left[E\left[T_{1}\right] E\left[T_{2}\right] \cdots E\left[T_{N}\right]\right]$, we can rewrite (10) in the following matrix form

$$
\boldsymbol{t}_{E}=\boldsymbol{u}+\boldsymbol{t}_{E} \boldsymbol{P} .
$$

Since $\boldsymbol{I}-\boldsymbol{P}$ is invertible [39], where $\boldsymbol{I}$ is the $N \times N$ identity matrix, all the average conditional vehicular sojourn times $E\left[T_{n}\right]$ for $1 \leq n \leq N$, required to compute the average sojourn time using the expression (9), can be obtained from

$$
\boldsymbol{t}_{E}=\boldsymbol{u}(\boldsymbol{I}-\boldsymbol{P})^{-1} .
$$

This completes the proof.

\subsection{Average Mobility Length}

Similar to the derivation of the average sojourn time, we can obtain the average vehicular mobility length, which is another key system metric related to the mobility behaviors and properties. First, we define $H_{n}$ as the conditional number of areas that a vehicle travels in the system, namely, the conditional vehicular mobility length, under the condition that it starts from area $A_{n}$, and denote the average value of $H_{n}$ over all the vehicles as $E\left[H_{n}\right]$. Further denote the vehicular mobility length in the system by $L$, and the average mobility length by $E[L]$. It can readily be seen that $E[L]$ is related to $E\left[H_{n}\right]$, $1 \leq n \leq N$, by

$$
E[L]=\sum_{n \in \mathcal{N}} p_{0 n} E\left[H_{n}\right] .
$$

The feasibility of computing $E[L]$ using the expression (12) is confirmed in the following theorem.

Theorem 2. Given the switching probability matrix $\boldsymbol{P}$ defined in (2), all the average conditional vehicular mobility lengths $E\left[H_{n}\right]$ for $1 \leq n \leq N$ are completely determined. Therefore, the average mobility length $E[L]$ can be computed using the expression (12).

Proof. Consider the average conditional mobility length conditioned on the vehicles starting from area $A_{n}$, namely, $E\left[H_{n}\right]$. It is easy to see that

$$
E\left[H_{n}\right]=1+\sum_{\substack{m \in \mathcal{N} \\ m \neq n}} p_{n m} E\left[H_{m}\right], 1 \leq n \leq N .
$$

Thus, all the average conditional mobility lengths, denoted as $\boldsymbol{h}_{E}=\left[E\left[H_{1}\right] E\left[H_{2}\right] \cdots E\left[H_{N}\right]\right]$, are obtained from

$$
\boldsymbol{h}_{E}=\mathbf{1}(\boldsymbol{I}-\boldsymbol{P})^{-1},
$$

where

$$
\mathbf{1}=\underbrace{\left[\begin{array}{lll}
1 & 1 & \cdots
\end{array}\right]}_{N} .
$$

This completes the proof.

\section{Model Validation}

Using the two largest available urban vehicular mobility traces described in Subsection 3.1, Shanghai and Beijing traces, we validate our proposed Markov jump process model. The traces record the location information of longitude and latitude of the vehicles during the trace collection periods. Therefore, we first need to process the two traces to fit our model. Then, we validate our model by using the empirical trace data, in terms of the following metrics: vehicular arrival rate, vehicular area distribution, average sojourn time and average mobility length.

\subsection{Time Selection and Area Partition}

In order to use the GPS vehicular trace to validate our model, we need to process the data on two different dimensions of time and location. In the time dimension, since a trace records the continuous mobility trajectory over the duration of whole day, we need to select an appropriate time observation period that is stable in terms of the number of vehicles observed in the system. In the location dimension, we need to partition the urban map into the areas according to the selected intersections, and then to decide which areas that the vehicles belong to according to their longitude and latitude information.

\subsubsection{Area Partition}

In order to divide the vehicular mobility system into the areas that vehicles transit between, we need to take the road and city structure into consideration. To focus on the downtown district, in Beijing city, we select the areas inside the 5th Ring Road as the modeled area and let the rest as the outside area; while in Shanghai city, we set Outer Ring Road as the boundary of the modeled area and outside area. As mentioned before, intersection is the most important factor in modeling the urban vehicular mobility and distributions. Therefore, we divide the system according to the positions of the key intersections in the city roads. More specifically, to obtain these key intersections, we consult the city map for the intersections connecting at least four-lanes of two-way roads by selecting them one by one, and then use the obtained key intersections as the centers of the partitioned areas, and employ the Voronoi diagram to achieve the actual partitioning. The Voronoi diagram is a frequently used method of decomposing a given space [37]. By employing the above approach, we divide the city into different areas according to the main road and city structures.

In a Voronoi diagram, one is given a finite set of sites $\mathcal{V}=\left\{v_{1}, v_{2}, \cdots, v_{N}\right\}$ in the Euclidean plane. The Voronoi cell $A_{n}$ corresponding to the site $v_{n}$ consists of all the points whose distances to $v_{n}$ are not greater than their distances to any other site $v_{m}, m \neq n$. In using the Voronoi diagram to partition the system region, the set of all the key intersections is $\mathcal{V}$. In other words, each site $v_{n}$ can be viewed as a selected intersection that is the center of the site or area. If we denote all the points in the system region to be modelled as $\mathcal{X}$, we can introduce the distance between the point $x \in \mathcal{X}$ and the site $v_{n} \in \mathcal{V}$ as $d_{x, v_{n}}=\left\|x-v_{n}\right\|$. The area $A_{n}$ for site $v_{n}$ can then be expressed formally as

$$
A_{n}=\left\{x \in \mathcal{X} \mid d_{x, v_{n}} \leq d_{x, v_{m}}, \forall v_{m} \in \mathcal{V} \backslash\left\{v_{n}\right\}\right\} .
$$


Based on this Voronoi diagram, we obtain all the boundaries that partition the system region into the $N$ different areas.

Taking the central part of Shanghai city as an example, the area partition result using the Voronoi diagram based partitioning method is shown in Fig. 2, where the blue dots are the records of vehicular trajectories, and the red points are the intersections selected to form the cells or areas which are separated by the yellow boundaries. After the region has been divided into the different areas, we can then decide which area a vehicle belongs to according to its longitude and latitude information. Consequently, the vehicular mobility is modelled by the transitions of vehicles from one area to another.

When processing the vehicular mobility trajectories, if a vehicle does not have record in any of the areas for the period of 10 minutes due to turning off, we assume that it has departed from the system, and will take it as a new vehicle moving into the system when it turns on and reappears. With the results of area partition, we derive the parameters of the model from the mobility traces. For each area $n$, related to the external arrival rate $\lambda_{n}$, we count the number of vehicles that come from the unmodeled areas and vehicles that re-appear in area $n$ due to turning on, and divide it by the time duration. Related to the probability of vehicles leaving the system $p_{n 0}$, we first count the number of vehicles moving out and turning off in area $n$, and then divided it by the number of the vehicles in area $n$ to obtained the leaving probability. Dividing the number of vehicles coming from other modeled areas by the number of vehicles in that area, we obtain the switching probability matrix $\mathbf{P}$. The expected vehicle residence time at area $n \mu_{n}$ is obtained by averaging the durations that the vehicles stay in area $n$. By the above described method, we get these parameters listed in Table 1 for the Beijing and Shanghai traces.

\subsubsection{Observation Period Selection}

We are interested in the period of time in the trace when vehicles are most active, or there is sufficient number of vehicles in the system, and the population of vehicles is relatively stable, or the number of vehicles in the system is approximately stationary. In an urban city, the vehicle traffic appears at almost the same patterns daily, except for some difference between a working day and weekend. Thus, we can select the suitable observation period from the trace by simply investigating the aggregate vehicular arrival rate in the time duration of one day, where the aggregate vehicular arrival rate is defined as the aggregated exogenous arrival rate that counts all the modeled areas and all vehicles in the system.

The aggregated vehicular arrival rates are plotted in Fig. 5 for Beijing and Shanghai traces, respectively. Observing the aggregated vehicular arrival rate curve of Beijing trace, the arrival rate is very low from the midnight to 5am, and it increases quickly during 6am to 9am, while the arrival rate almost keeps in the same high level during the daytime from 9am to 7pm, and this is followed by a rate-decreasing period lasting into the midnight. Similar arrival rate patterns can be observed in the Shanghai trace, except that the traffic of Shanghai is still in a very active

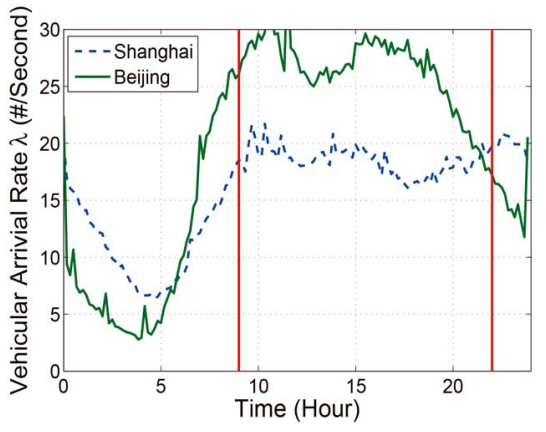

Fig. 5. Average vehicular arrival rate to the system as function of time in the one-day duration for Shanghai and Beijing traces.

state during the period of 7pm to well over the midnight. This clearly reflects a much higher level of night-time life in Shanghai than in Beijing. Combing the results for the both traces, we select the period of 9am to $7 \mathrm{pm}$ as the observation period for the trace data to validate our proposed model. Under this period selection, we set 9am and 7pm as the starting time and the ending of time of the arrival events of one day respectively, and ignore the arrival events that across two days since these obviously should not be considered as an arrival sample.

\subsubsection{Training Trace Selection}

In order to validate our proposed mobility model by a persuasive approach, we train the model with only part of the trace, and then use the trained model to describe the rest part of the trace. Specifically, for the used Shanghai and Beijing trace respectively, we split the trace into two parts with equal time duration. For the first half of the trace, we treat it as the "training data", which is used for training the proposed model to obtain the model parameters. While for the second half of the trace, we treat it as "validation data", and use it to validate the model obtained from the "training data" by comparing the results calculated from the model with that obtain from the trace. By this usually used approach of model validation in practice, we validate our model in terms of vehicular area distribution, average sojourn time and mobility length.

\subsection{Arrival Rate Validation}

An important requirement for our proposed mobility model is that the arrival to each area should follow a Poisson process. Therefore, it is necessary to validate that this assumption holds for the vehicular mobility system. In the Markov jump process, the arrival to each states is the aggregated customer arrival process. Consequently, the aggregated arrival is the metric for validating our proposed model. We validate the vehicular arrival rate in the time scale of all days as well as in the time scale of one day.

Firstly, we investigate the aggregated effective arrival of all the vehicles in the system. After dividing the system into areas based on the area partitioning method discussed in previous subsection, we count the effective arrivals to each area. To validate the aggregated arrival rate follows a Poisson process, we need to show that the inter-arrival duration can be fitted well by the exponential distribution. We plot the empirical complementary cumulative 


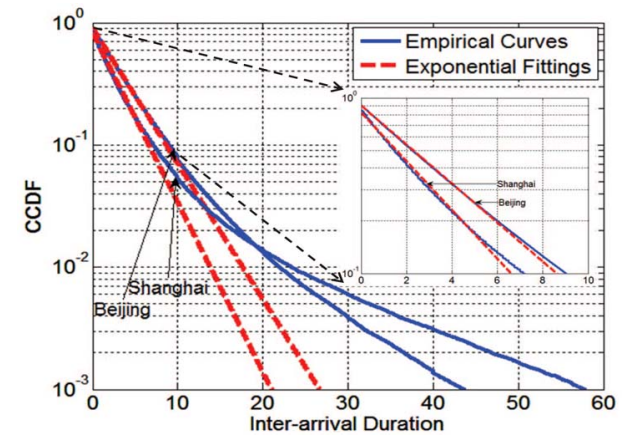

Fig. 6. Comparison of the empirical distributions of aggregated effective inter-arrival duration, which includes both the exogenous and endogenous arrivals, with the exponential distribution fittings for Shanghai and Beijing trace.

distribution functions (CCDFs) of the inter-arrival duration obtained from Shanghai and Beijing traces, respectively, in Fig. 6, where we also use an exponential distribution to fit each of the two empirical distributions for comparison. The values of CCDF are in the range of $1(100 \%)$ to $0(0 \%)$. Since inter-arrival duration distribution has a longer tail as shown in Fig. 6, we focus on the range of CCDF values from 1 to 0.1 , i.e., the $90 \%$ of the distribution. Since we plot the results in the linear-log scale, the curve of an exponential distribution becomes a straight line, as can be seen for the two dashed curves of the fitted exponential distributions for Shanghai and Beijing trace data. We observe clearly from Fig. 6 that the empirical inter-arrival time distributions of the two traces match the corresponding two fitted exponential distributions well. The goodness of fit is further measured quantitatively by the R-square statistics [40], which is defined as the percentage of the variation between the empirical CCDF and the fitted distribution. The average adjusted R-square statistics are over $98 \%$ for both Shanghai and Beijing traces. Thus, these results demonstrate that the exponential distribution fits most of the inter-arrival distribution. This confirms the accuracy of the exponential distributed aggregated inter-arrival duration, which indicates that the aggregated effective arrivals follow a Poisson process.

Secondly, in order to further validate that the exogenous arrival rate to each area follows a Poisson distribution, we investigate the distribution of the exogenous arrival time of each area in the time scale of one day, and select the first 15 days of the month for comparison. We use the Kolmogorov Smirnov (KS) test to measure the closeness of the Poisson distribution and empirical one, instead of CCDF display, to overcome the difficulty of displaying a large amount of distribution curves in each area of 15 days. The KS statistic can quantify the distance between the empirical distribution function of the sample and the theoretically fitted distribution function [41]. The smaller the KS statistic, the closer the two distributions are. In our study, we set the significance level [41] of the KS test to 0.01, which indicates the confidence level of $99 \%$. Fig. 7 shows the goodness of fit measured by the acceptance ratio of the KS tests for each day by averaging the results over all the areas. From the results shown in Fig. 7, we observe that the acceptance ratios of the KS test for Beijing trace are above 90\% except

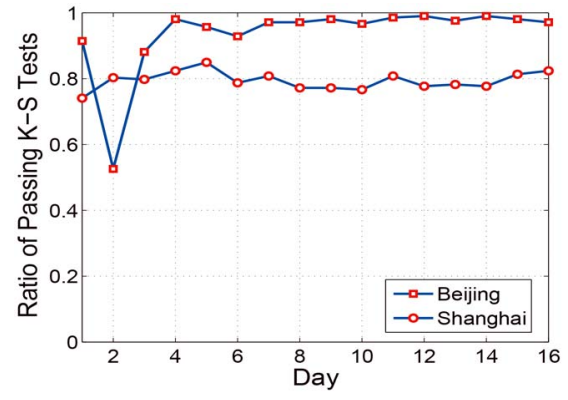

Fig. 7. Ratios of passing Kolmogorov Smirnov tests for the vehicular arrival rate in the time scale of one day for Shanghai and Beijing traces.

for the second day, which turns out to have a relatively smaller amount of vehicle mobility records since that day was the national Labor's holiday for the city and hence much less traffics on the street. For Shanghai trace, we also note a good match between the model distribution and the empirical result, as the average acceptance ratios of the KS test are around $80 \%$. Combing the results of Shanghai and Beijing traces, which indicate the overall accuracy of the Poisson model is above $80 \%$, we conclude that the exogenous arrivals to each area can be accurately modelled by a Poisson distribution.

The above results of arrival rate validation clearly demonstrate the accuracy of the Poisson distribution for exogenous and effective arrivals in our model. We now turn our attention to compare the vehicular distribution, sojourn time and mobility length predicted or calculated using the Markov jump process with superimposed Poisson arrival based vehicular mobility model with the empirical results obtained directly from the two traces.

\subsection{Vehicular Distribution}

We investigate the single area vehicular distribution by comparing the results obtained from the proposed theoretical model and those based directly on the empirical data. Firstly, by selecting the six busiest areas from Beijing and Shanghai traces, we estimate the parameters of the proposed model from the traces, of which the most important parameters $\gamma_{n}, 1 / \mu_{n}$ and $\rho_{n}$ are shown in Tables 2 and 3 respectively. we plot the empirical distribution of the number of vehicles in each area as well as the theoretical single area vehicular distribution calculated according to Lemma 1. The results for Beijing trace are shown in Fig. 8, while the results for Shanghai are depicted in Fig. 9, where the solid curves are the model based single area vehicular distributions, and the dashed curves are the empirical results. We observe that the model predicted vehicular distribution matches well the empirical distribution directly obtained from the trace data. This demonstrates the accuracy of our

TABLE 2

Parameters of the Model Estimated from the Beijing Trace for the Selected Six Intersections ('IS' Denotes 'Intersection')

\begin{tabular}{c|cccccc}
\hline Parameters & IS 1 & IS 2 & IS 3 & IS 4 & IS 5 & IS 6 \\
\hline$\gamma_{n}$ & 0.026 & 0.025 & 0.024 & 0.022 & 0.022 & 0.022 \\
$1 / \mu_{n}$ & 129.27 & 102.28 & 178.82 & 113.81 & 155.83 & 125.00 \\
$\rho_{n}$ & 3.40 & 2.60 & 4.35 & 2.51 & 3.39 & 2.74 \\
\hline
\end{tabular}


TABLE 3

Parameters of the Model Estimated from the Shanghai Trace for the Selected Six Intersections ('IS' Denotes 'Intersection')

\begin{tabular}{c|cccccc}
\hline Parameters & IS 1 & IS 2 & IS 3 & IS 4 & IS 5 & IS 6 \\
\hline$\gamma_{n}$ & 0.095 & 0.074 & 0.072 & 0.068 & 0.068 & 0.063 \\
$1 / \mu_{n}$ & 47.26 & 104.88 & 63.81 & 54.91 & 52.41 & 44.78 \\
$\rho_{n}$ & 4.48 & 7.81 & 4.56 & 3.73 & 3.54 & 2.80 \\
\hline
\end{tabular}

proposed Markov jump process based vehicular mobility model.

On the other hand, as shown in Figs. 8 and 9, there still exists some deviations between the model and empirical results. The reason is that the model distribution is obtained by the theoretical calculation of related parameters that are statistical from the traces. In some of the intersections' vehicular distribution, the deviations in the peak become as large as $15 \%$. However, it is difficult and biased to access the deviation only in the peak point. In order to measure the average closeness of the model predicted distributions to the empirical distributions for all the areas, we use the model distribution predicted by Lemma 1 to fit the empirical vehicular distribution curve for each of all the areas. The goodness of fit for the distribution of each area is also measured quantitatively by the R-square statistics [40]. After obtaining all the R-square statistics for all the areas for both Shanghai and Beijing traces, respectively, we plot the two aggregated R-square distributions, i.e. the two CCDFs of all the R-square statistics for all the areas for the two traces in Fig. 10. It can be seen from Fig. 10 that the average adjusted R-square statistics of over $80 \%$ areas is larger than $90 \%$ for Beijing trace, while the average adjusted R-square statistics of over $92 \%$ areas is larger than $90 \%$ for Shanghai trace. This further confirms the accuracy of the model-based prediction for vehicular distribution.

\subsection{Average Sojourn Time and Mobility Length}

We compare the theoretical results of mean sojourn time and mobility length predicted from our proposed model according to Theorems 1 and 2 with the empirical results obtained directly from the two mobility traces. To test the scalability and accuracy of the proposed model, we vary the number of vehicles involved in the investigation. For both Shanghai and Beijing traces, respectively, we sort the vehicles according to their numbers of positions recorded in the GPS trace. We first select the vehicles which are recorded in the
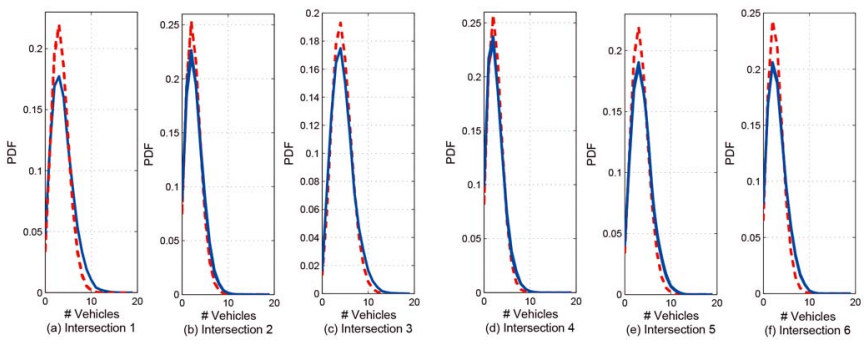

Fig. 8. Vehicular distributions of six intersections for Beijing trace, where the dashed curves are the empirical results obtained from the trace, while the solid curves are the theoretical results calculated by our proposed model.
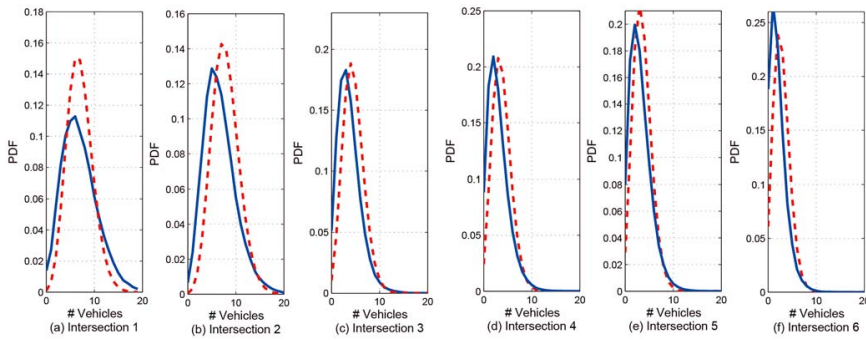

Fig. 9. Vehicular distributions of six intersections for Shanghai trace, where the dashed curves are the empirical results obtained from the trace, while the solid curves are the theoretical results calculated by our proposed model.

trace at least $80 \%$ of the trace collection time, and then further increase the number of vehicles involved. Specifically, for Shanghai trace, we select the three different numbers of vehicles as: 1000 (these vehicles have $80 \%$ of the records in the trace), 3000 and 4441, while for Beijing trace, we set the four different numbers of vehicles as: 3000 (these vehicles have $80 \%$ of the records in the trace), 6000, 10000 and 28590. For these different settings, since the number of vehicular changes, the system parameters such as exogenous arrival rate and effective arrival rate should be also different. Thus, for each set of the vehicles, we re-generate the model by obtaining the new parameters that are best fitting with the subset of the selected vehicles, and then obtain the results of average sojourn time and mobility length from the built model.

The results of the model predicted average sojourn time and mobility length are compared with the corresponding empirical results obtained directly from the trace data in Tables 4 and 5 for Shanghai and Beijing traces, respectively. For the average sojourn time, we observe from Tables 4 and 5 that the model predicted results match well the empirical results, where the accuracy of a model predicted result is quantified by the deviation measure given by

$$
\text { Deviation }=\frac{\mid \text { Predicted }- \text { Empirical } \mid}{\text { Empirical }} \times 100 \% .
$$

In terms of average mobility length, we can see from Tables 4 and 5 that the model predicted results also match the empirical results very well. Thus, we can conclude that our proposed model is sufficiently accurate to model the

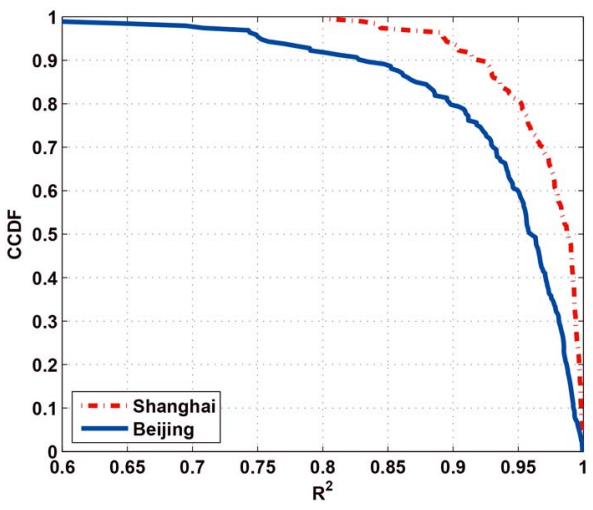

Fig. 10. Distribution of the adjusted R-square statistics for the theoretical fittings of the empirical vehicular distribution curves. 
TABLE 4

Model Predicted and Empirical Results of Average Sojourn Time and Mobility Length for Shanghai Trace

\begin{tabular}{c|ccc|ccc}
\hline Mobility & \multicolumn{3}{|c|}{ Average Sojourn Time } & \multicolumn{3}{c}{ Average Mobility Length } \\
Number of Vehicles & Predicted & Empirical & Deviation & Predicted & Empirical & Deviation \\
\hline 1000 & 1526.9 & 1348.5 & $11.6 \%$ & 16.48 & 16.91 & $2.6 \%$ \\
3000 & 1296.4 & 1197.3 & $7.6 \%$ & 11.22 & 11.45 & $2.1 \%$ \\
4441 & 1323.2 & 1186.1 & $10.4 \%$ & 11.53 & 11.43 & $1.0 \%$ \\
\hline
\end{tabular}

TABLE 5

Model Predicted and Empirical Results of Average Sojourn Time and Mobility Length for Beijing Trace

\begin{tabular}{c|ccc|ccc}
\hline Mobility & \multicolumn{3}{|c|}{ Average Sojourn Time } & \multicolumn{3}{c}{ Average Mobility Length } \\
Number of Vehicles & Predicted & Empirical & Deviation & Predicted & Empirical & Deviation \\
\hline 3000 & 1606.9 & 1680.0 & $4.5 \%$ & 9.55 & 9.18 & $3.9 \%$ \\
6000 & 1598.8 & 1672.9 & $4.6 \%$ & 9.38 & 9.00 & $4.1 \%$ \\
10000 & 1596.6 & 1670.7 & $4.6 \%$ & 9.37 & 9.02 & $3.8 \%$ \\
28590 & 1435.0 & 1500.6 & $4.6 \%$ & 8.49 & 8.15 & $4.0 \%$ \\
\hline
\end{tabular}

vehicular mobility and it can be utilized to evaluate the vehicular mobility system's performance.

\subsection{Discussion}

In the above model validation, we have verified that our proposed macroscopic-level vehicular mobility is able to describe the complex global vehicular mobility and predict various key measures regarding steady-state and networklevel performance, such as vehicular distribution, average sojourn time, and mobility length in the scale of a city. Although this macroscopic-level model describes the overall mobility of the whole network in the steady state, it does not have the capability to describe individual mobility behaviors, interactions between vehicles, and time-variant properties precisely. That is to say, our macroscopic-level mobility model does not capture the microscopic individual vehicular mobility related features such as mobility direction, flight length, pause time, vehicle-to-vehicle connectivity, etc. On the other hand, our model also does not capture the time-variant properties according to other means of traffic control such as gate-control signal for freeway or the time-variant traffic congestions. Indeed, it captures the average service time for the modeled areas to describe the spatial traffic distribution of a city. Thus, in order to provide a comprehensive vehicular mobility model, we should take both the macroscopic and microscopic level mobility parameters together into consideration in the future work.

\section{Applications to Networking}

In this section, we use two applications as examples to illustrate the effectiveness of our proposed urban vehicular mobility model in the analysis of the system-level performance and dimensioning for vehicular networks.

\subsection{RSU Capacity Decision}

In a vehicular network, data traffics initiated from vehicles are random and bursty by nature. RSUs act as the gateways to the Internet and to other infrastructure systems such as ITS. Vehicles transmit their Internet access requests and information to RSUs, and RSUs then send responses to the Internet for querying the data and information needed by vehicles. How RSUs are deployed critically influences the performance of a vehicular network, since the capacity and quantity of the deployed RSUs determine the capacity and service that can be provided by the vehicular network. Deploying a large number of RSUs to offer large capacity, however, incurs a huge infrastructure cost. Therefore, the decision for RSU deployment should depend on the demands of the vehicular network. In a large urban city, it is very difficult to make such decision because of the dynamics of vehicular traffic and the randomness of vehicular mobility. However, based on our proposed vehicular model, we can obtain some fundamental results for the relationship between the RSUs' capacity and the network performance. Using the proposed Markov jump process based vehicular mobility model, we now analyze how much the capacity of the RSUs should provide with the increase of the communication demands as the consequence of the increasing number of vehicles in the system.

The vehicular traffic and communication traffic in a vehicular system are both dynamic. Roughly speaking, the capacity of an RSU is the maximum number of vehicles that the RSU is able to serve. We may define that an RSU is overloaded if the time period, in which the number of vehicles requiring service is more than its capacity, exceeds $95 \%$ of the whole measurement time. For analysis purpose, we will make the assumptions that each area of the system has one RSU and all the RSUs have the same capacity. The vehicular system may be regarded as overloaded when the certain percentage of the RSUs are overloaded. In order to proceed the investigation, we need to define the average exogenous arrival rate per area, denoted as $\lambda$, which is the average of all the area exogenous arrival rates $\lambda_{n}$, for $1 \leq n \leq N$. Alternatively, all the areas $A_{n}$ may be assumed to have the same exogenous arrival rates $\lambda_{n}=\lambda$, for $1 \leq n \leq N$. By increasing the average exogenous arrival rate per area $\lambda$ in our model, we investigate the fraction of the RSUs that will become overloaded as the number of vehicles entering the system increases.

From both Shanghai and Beijing traces, respectively, we select the vehicles which have most completely records during the whole trace collection time, the number of which is 3000 for the both traces. We use these 3000 taxis to obtain the system's average exogenous arrival rate per area $\lambda$ from each trace. The vehicular arrival rate of Beijing trace is 


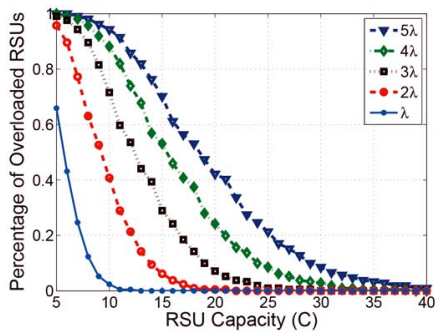

Fig. 11. Percentage of the overloaded RSUs versus the RSU capacity as the function of the vehicular arrival rate for Beijing trace.

obviously different from that of Shanghai trace. We then change the average exogenous arrival rate per area from $\lambda$ to $5 \lambda$ in our model, and plot the percentage of the overloaded RSUs as the function of the RSU capacity for each given average exogenous arrival rate per area. The results so obtained are depicted in Figs. 11 and 12 for Beijing and Shanghai traces, respectively. From the results shown in Figs. 11 and 12, we observe that the fraction of the overloaded RSUs decrease very rapidly as the RSU capacity increases and, not surprisingly, the percentage of the overloaded RSUs increase with the increase of vehicles entering the system.

In particular, we examine the RSU capacity required to ensure that at least $95 \%$ of the RSUs are not overloaded. The results are summarized in Tables 6 and 7 for Beijing and Shanghai traces, respectively. For Beijing trace, we note that when the vehicular arrival rate is $\lambda$, the required capacity is about 10, while when the arrival rate is increased by 5 times to $5 \lambda$, the required RSU capacity is about 35 , which only increases 3.5 times. For Shanghai trace, we have similar observation when the vehicular arrival rate is increased from $\lambda$ to $5 \lambda$. It can be seen that the RSU capacity does not need to increase linearly with the number of vehicles in the system. The investigation here demonstrates the potential of applying our vehicular mobility model to design the RSU deploying policy according to the network performance requirements and the costs of RSU equipments.

\subsection{Combined V2I and V2V Performance Analysis}

In reality, it is difficult and uneconomical to cover the roads of an urban city with sufficiently many RSUs so that each vehicle on road can always be connected to a nearby RSU. Instead, vehicle-to-vehicle (V2V) communications by opportunistic contacts offers higher bandwidth communication capacity for data transmission, which can be exploited to form what is known as the opportunistic vehicular network or vehicular delay tolerant network

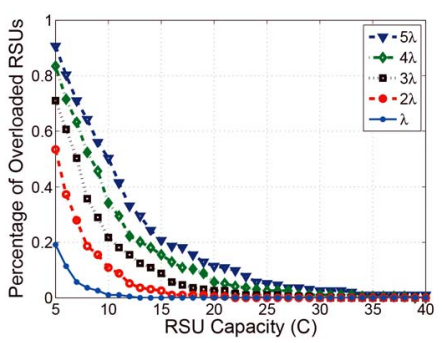

Fig. 12. Percentage of the overloaded RSUs versus the RSU capacity as the function of the vehicular arrival rate for Shanghai trace.
TABLE 6

The RSU Capacity Required to Ensure that at Least $95 \%$ of the RSUs are not Overloaded for Beijing Trace

\begin{tabular}{|c|ccccc|}
\hline Average arrival rate per area & $\lambda$ & $2 \lambda$ & $3 \lambda$ & $4 \lambda$ & $5 \lambda$ \\
\hline Roadside unit capacity & 10 & 16 & 22 & 29 & 35 \\
\hline
\end{tabular}

(VDTN) [42]. By exploiting the delay-tolerant nature of non-realtime applications, service providers can delay and even shift the data transmission to a VDTN. Therefore, in vehicular networking, vehicle to RSUs (V2I) communications and V2V communications are usually combined to offer more effective services. As the second application, we now study the use of our proposed mobility model to investigate the performance of the combined V2I and V2V communications. We demonstrate that, based on the proposed mobility model, we can derive the closed-form expressions of the key performance metrics for the combined V2I and V2V communication system, such as the probability of the network-wide service satisfaction and the average number of the areas that meet the communication demands. Roughly speaking, an area is the service satisfactory area if every vehicle in this area can receive the data at the rate of its communication requirement. A network is in the state of communication service satisfaction if all the vehicles in the network are satisfied. Obviously, for a vehicular network, it is very hard for the network to enjoy the communication service satisfaction state all the time. Therefore, the steady-state probability that the network is in the state of communication service satisfaction is a key metric to evaluate the performance of a vehicular network. Another important metric is the expected number of areas that are enjoying communication service satisfaction. We now give the more precise definitions for these two metrics.

The communication capacity index for area $A_{n}$, denoted by $\Phi_{n}\left(W_{n}\right)$, is defined as

$$
\Phi_{n}\left(W_{n}\right)=\frac{c_{n}+p_{n}}{d_{n}\left(W_{n}\right)},
$$

where $W_{n}$ is the number of vehicles in area $A_{n}, c_{n}$ is the communication capacity of the deployed RSU in $A_{n}, p_{n}$ denotes the capacity of the $\mathrm{V} 2 \mathrm{~V}$ communication in $A_{n}$, and $d_{n}\left(W_{n}\right)$ is the communication demand of the vehicles in $A_{n}$. Based on $\Phi_{n}\left(W_{n}\right)$, the probability that area $A_{n}$ is enjoying communication service satisfaction can be defined as

$$
A S_{n}=P\left(\Phi_{n}\left(W_{n}\right) \geq 1\right) .
$$

The probability that all the areas are enjoying communication service satisfaction is then defined by

$$
\begin{aligned}
P S & =P\left(\Phi_{1}\left(W_{1}\right) \geq 1, \Phi_{2}\left(W_{2}\right) \geq 1, \ldots, \Phi_{N}\left(W_{N}\right) \geq 1\right) \\
& =\prod_{n=1}^{N} P\left(\Phi_{n}\left(W_{n}\right) \geq 1\right)=\prod_{n=1}^{N} A S_{n} .
\end{aligned}
$$

TABLE 7

The RSU Capacity Required to Ensure that at Least $95 \%$ of the RSUs are not Overloaded for Shanghai Trace

\begin{tabular}{|c|ccccc|}
\hline Average arrival rate per area & $\lambda$ & $2 \lambda$ & $3 \lambda$ & $4 \lambda$ & $5 \lambda$ \\
\hline Roadside unit capacity & 8 & 13 & 17 & 22 & 26 \\
\hline
\end{tabular}


On the other hand, the average number of the areas that enjoy communication service satisfaction can be defined as

$$
N S=\sum_{n=1}^{N} A S_{n}=\sum_{n=1}^{N} P\left(\Phi_{n}\left(W_{n}\right) \geq 1\right) .
$$

We now calculate the probability of area $A_{n}$ enjoying communication service satisfaction, i.e. $A S_{n}$. Assume that a vehicle $i \in \mathcal{W}_{n}$ can offer the capacity of $u_{i}$, where $\mathcal{W}_{n}$ denotes the index set of the vehicles that are in area $A_{n}$, which is conceptually given by

$$
\mathcal{W}_{n}=\left\{0,1,2, \ldots, W_{n}\right\}
$$

Thus, $p_{n}=\sum_{i \in \mathcal{W}_{n}} u_{i}$. Further assume that each vehicle in area $A_{n}$ need the communication capacity of $r_{n}$. Then $d_{n}\left(W_{n}\right)=W_{n} r_{n}$. Therefore, $A S_{n}$ can be expressed as

$$
A S_{n}=P\left(c_{n}+\sum_{i \in \mathcal{W}_{n}} u_{i} \geq W_{n} r_{n}\right) .
$$

In a real vehicular system, the $\mathrm{V} 2 \mathrm{~V}$ communication capacity will depend on the wireless interfaces adopted. For an illustrative purpose, suppose that there are two classes of vehicles in the system, and class-one vehicles use BlueTooth, which has a low capacity of $u^{\text {bt }}$, while class-two vehicles use WiFi, which has a large capacity of $u^{\mathrm{wf}}$, to achieve the short range peer to peer communications. Let the number of BlueTooth vehicles in area $A_{n}$ be $W_{n}^{\mathrm{bt}}$ and the number of WiFi vehicles in $A_{n}$ be $W_{n}^{\mathrm{wf}}$. Then $W_{n}^{\mathrm{bt}}+W_{n}^{\mathrm{wf}}=W_{n}$, and

$$
\mathcal{W}_{n}=\mathcal{W}_{n}^{\mathrm{bt}} \bigcup \mathcal{W}_{n}^{\mathrm{wf}},
$$

where $\mathcal{W}_{n}^{\mathrm{bt}}$ and $\mathcal{W}_{n}^{\mathrm{wf}}$ are the index sets of the vehicles in $A_{n}$ that employ BlueTooth and WiFi for V2V communications, respectively. The sizes of $\mathcal{W}_{n}^{\mathrm{bt}}$ and $\mathcal{W}_{n}^{\mathrm{wf}}$ are obviously $W_{n}^{\mathrm{bt}}$ and $W_{n}^{\mathrm{wf}}$, respectively. We also need to introduce the two types of loads in area $A_{n}, \rho_{n}^{\text {bt }}$ and $\rho_{n}^{\text {wf }}$. According to Lemma $1, \rho_{n}^{\text {bt }}$ is the expected number of BlueTooth vehicles in area $A_{n}$, while $\rho_{n}^{\mathrm{wf}}$ is the expected number of WiFi vehicles in $A_{n}$.

In order to enable theoretical analysis and derive explicit performance results, we neglect the different properties between BlueTooth and WiFi communications in terms of contact patterns and commination duration, and also do not take the BlueTooth-WiFi contacts into the considerations. With the above introduced notations and approximations, the probability of area $A_{n}$ enjoying communication service satisfaction can be approximately expressed as

$$
\begin{array}{r}
A S_{n} \doteq P\left(c_{n}+W_{n}^{\mathrm{bt}} u^{\mathrm{bt}}+W_{n}^{\mathrm{wf}} u^{\mathrm{wf}} \geq\left(W_{n}^{\mathrm{bt}}+W_{n}^{\mathrm{wf}}\right) r_{n}\right) \\
=\sum_{w_{n}^{\mathrm{bt}} \in \mathcal{W}_{n}^{\mathrm{bt}}} \sum_{n}^{\mathrm{wf} \in \mathcal{W}_{n}^{\mathrm{wf}}} P\left(W_{n}^{\mathrm{bt}}=w_{n}^{\mathrm{bt}}, W_{n}^{\mathrm{wf}}=w_{n}^{\mathrm{wf}}\right) \times \\
P\left(\frac{c_{n}+W_{n}^{\mathrm{bt}} u^{\mathrm{bt}}+W_{n}^{\mathrm{wf}} u^{\mathrm{wf}}}{W_{n}^{\mathrm{bf}}+W_{n}^{\mathrm{wf}}} \geq r_{n} \mid W_{n}^{\mathrm{bt}}=w_{n}^{\mathrm{bt}}, W_{n}^{\mathrm{wf}}=w_{n}^{\mathrm{wf}}\right) \\
=\sum_{w_{n}^{\mathrm{bt}} \in \mathcal{W}_{n}^{\mathrm{bt}}} \sum_{n}^{\mathrm{wf} \in \mathcal{W}_{n}^{\mathrm{wf}}} P\left(W_{n}^{\mathrm{bt}}=w_{n}^{\mathrm{bt}}\right) P\left(W_{n}^{\mathrm{wf}}=w_{n}^{\mathrm{wf}}\right) \\
\quad \times 1\left(c_{n}+u^{\mathrm{bt}} w_{n}^{\mathrm{bt}}+u^{\mathrm{wf}} w_{n}^{\mathrm{wf}} \geq\left(w_{n}^{\mathrm{bf}}+w_{n}^{\mathrm{wf}}\right) r_{n}\right) \\
=\sum_{w_{n}^{\mathrm{bt}} \in \mathcal{W}_{n}^{\mathrm{bt}}} \sum_{n}^{\mathrm{wf} \in \mathcal{W}_{n}^{\mathrm{wf}}} P\left(W_{n}^{\mathrm{bt}}=w_{n}^{\mathrm{bt}}\right) P\left(W_{n}^{\mathrm{wf}}=w_{n}^{\mathrm{wf}}\right)
\end{array}
$$

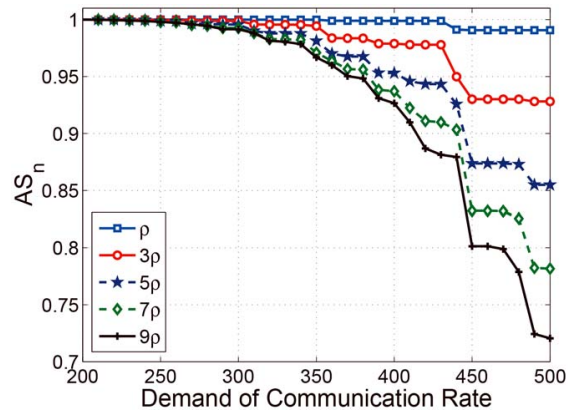

Fig. 13. Service satisfaction probability of the most loaded area versus the demand of communication rate, given various average area loads.

$$
\begin{aligned}
& \times 1\left(\left(r_{n}-u^{\mathrm{bt}}\right) w_{n}^{\mathrm{bt}}+\left(r_{n}-u^{\mathrm{wf}}\right) w_{n}^{\mathrm{wf}} \leq c_{n}\right)
\end{aligned}
$$

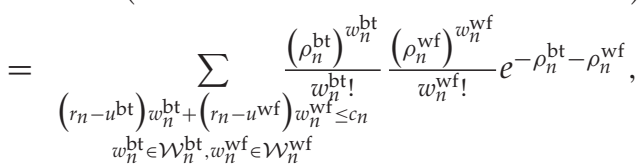

where 1 ("expression") is the indicator function that equals to 1 if the "expression" is true, and otherwise equals to 0 , while the last equation is obtained according to Lemma 1. After obtaining $A S_{n}$ for $1 \leq n \leq N$, the probability of the network-wide communication service satisfaction, $P S$, and the average number of communication service satisfaction areas, NS, can be obtained by substituting $A S_{n}$ of (22) for all $n$ into (17) and (18), respectively.

Based on the above derivation, we set up a vehicular network environment to observe the performance. We assume that there are 20 areas in this simulated vehicular network, and the RSU's communication capacity of each area, namely, $c_{n}$ of area $A_{n}$, is set to be uniformly distributed in [500, 12000] bps, which reflects the capacity provided by usually deployed WiFi or 3G/4G stations. For V2V communications, $u^{\text {bt }}$ of each low-capacity vehicle is randomly and uniformly chosen from [50, 150] bps, while $u^{\mathrm{wf}}$ of each high-capacity vehicle is randomly and uniformly chosen from $[750,850]$ bps. We set the loads $\rho_{n}$ of the areas $A_{n}$, $1 \leq n \leq 20$, to those of the 20 areas in Beijing trace, which have the largest numbers of vehicles in the trace data, and we further let half of the vehicles in each area have the low V2V capacity and the other half have the high V2V capacity, namely, $\rho_{n}^{\mathrm{bt}}=\rho_{n}^{\mathrm{wf}}=\frac{1}{2} \rho_{n}$. For analysis purpose, we define the average area load as $\rho$, which is simply the average of all the $\rho_{n}$ for $1 \leq n \leq 20$. Again for analysis purpose, all the vehicles have the same communication demand, which follows the exponential distribution with parameter $\vartheta$. The mean of the communication demand of vehicles is therefore $1 / \vartheta$.

In the simulation study, we start with the average area load to be $\rho$, and then increase it to 3, 5, 7 and 9 times. We observe the performance of the vehicular networking system as the function of the demand of communication rate $1 / \vartheta$ for the given average area load, in terms of the area service satisfaction probability $A S_{n}$, the network-wide satisfaction probability $P S$ and the average number of the service satisfaction areas NS. Fig. 13 depicts the results of the service satisfaction probability for the most loaded area in the system. It can be seen from Fig. 13 that the communication service satisfaction probability is near $100 \%$ when the 


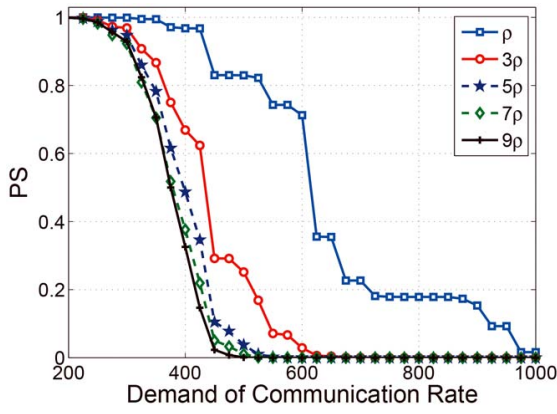

Fig. 14. Network-wide service satisfaction probability versus the demand of communication rate, given various average area loads.

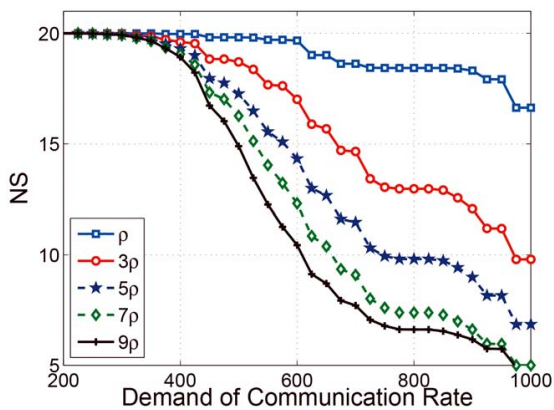

Fig. 15. Average number of the communication service satisfaction areas versus the demand of communication rate, given various average area loads.

average demand is less than $300 \mathrm{bps}$. With the increase of the demand, the service satisfaction probability decreases. Furthermore, the larger the load, the sharper the decreasing rate in the communication service satisfaction probability. These results provide the planner with useful information in deciding how to deploy the RSU device to meet the specific performance requirements. In terms of the networkwide performance, Figs. 14 and 15 shows the results of the network-wide satisfaction probability and the average number of the service satisfaction areas, respectively. It can be seen from Figs. 14 and 15 that the network-wide performance degrades with the increase in the average demand and area load. Again, these results offer useful assistance to the combined V2I and V2V network design.

\section{CONCLUSION}

In this paper, we have used the Markov jump process to model the macroscopic-level vehicular mobility. Based on the two large-scale urban city vehicular motion traces, Shanghai and Beijing traces, we have validated the accuracy of our proposed model. Moreover, we have demonstrated that this proposed simple model can accurately describe the complex vehicular mobility and predict various key measures of the network-level and vehicular-level performance. Furthermore, we have used the two applications, determining the RSU capacity and analysing the combined V2I and $\mathrm{V} 2 \mathrm{~V}$ network performance, to illustrate the effectiveness of our proposed model in the analysis of system-level performance and dimensioning for vehicular networks. On the other hand, our proposed vehicular mobility model does not capture the properties of time-variant and behaviors of individual mobility, which will be covered by our future work through investigating how to integrate both the macroscopic and microscopic level mobility parameters together.

\section{ACKNOWLEDGMENTS}

This work is supported by National Basic Research Program of China (973 Program) (2013CB329105), National Nature Science Foundation of China (61301080, 61171065, and 61273214), National High Technology Research and Development Program (2013AA013501 and 2013AA013505), Chinese National Major Scientific and Technological Specialized Project (2013ZX03002001), and China's Next Generation Internet (CNGI-12-03-007).

\section{References}

[1] M. Khabazian, S. Aissa, and M. Mehmet-Ali, "Performance modeling of message dissemination in vehicular ad hoc networks with priority," IEEE J. Sel. Areas Commun., vol. 29, no. 1, pp. 61-71, Jan. 2011.

[2] G. Dimitrakopoulos and P. Demestichas, "Intelligent transportation systems," IEEE Veh. Technol. Mag., vol. 5, no. 1, pp. 77-84, Mar. 2010.

[3] J. Zhao and G. Cao, "VADD: Vehicle-assisted data delivery in vehicular ad hoc networks," in Proc. 25th IEEE INFOCOM, Barcelona, Spain, Apr. 2006, pp. 1-12.

[4] F. Li and Y. Wang, "Routing in vehicular ad hoc networks: A survey," IEEE Veh. Technol. Mag., vol. 2, no. 2, pp. 12-22, Jun. 2007.

[5] A. Abdrabou and W. Zhuang, "Probabilistic delay control and road side unit placement for vehicular ad hoc networks with disrupted connectivity," IEEE J. Sel. Areas Commun., vol. 29, no. 1, pp. 129-139, Jan. 2011.

[6] J. Harri, F. Filali, and C. Bonnet, "Mobility models for vehicular ad hoc networks: A survey and taxonomy," IEEE Commun. Surv. Tutor., vol. 11, no. 4, pp. 19-41, Dec. 2009.

[7] D. Helbing, "Traffic and related self-driven many-particle systems," Rev. Mod. Phys., vol. 73, no. 4, pp. 1067-1141, 2001.

[8] Y.-C. Chen, J. Kurose, and D. Towsley, "A simple queueing network model of mobility in a campus wireless network," in Proc. 3rd ACM Workshop Wireless of the Students, by the Students, for the Students, Las Vegas, NV, USA, Sep. 2011, pp. 5-8.

[9] L. Breuer, From Markov Jump Proceses to Spatial Queues. Boston, MA, USA: Kluwer Academic Pubulication, 2003.

[10] H. Abu-Ghazaleh and A. S. Alfa, "Application of mobility prediction in wireless networks using Markov renewal theory," IEEE Trans. Veh. Technol. , vol. 59, no. 2, pp. 788-802, Feb. 2010.

[11] W.-Y. Lee and I. F. Akyildiz, "Spectrum-aware mobility management in cognitive radio cellular networks," IEEE Trans. Mobile Comput., vol. 11, no. 4, pp. 529-542, Apr. 2012.

[12] P. Fazio and S. Marano, "Mobility prediction and resource reservation in cellular networks with distributed Markov chains," in Proc. 8th Wireless Commun. Mobile Comput. Conf., Limassol, Cyprus, Aug. 2012, pp. 878-882.

[13] D. Wang and A. A. Abouzeid, "On the cost of knowledge of mobility in dynamic networks: An informationtheoretic approach," IEEE Trans. Mobile Comput., vol. 11, no. 6, pp. 995-1006, Jun. 2012.

[14] R. R. Roy, "Random walk mobility," in Handbook of Mobile Ad Hoc Networks for Mobility Models. New York, NY, USA: Springer, 2011, pp. 35-63.

[15] J. Yoon, M. Liu, and B. Noble, "Random waypoint considered harmful," in Proc. IEEE INFOCOM , San Francisco, CA, USA, Apr. 2003, pp. 1312-1321.

[16] A. Rojas, P. Branch, and G. Armitage, "Experimental validation of the random waypoint mobility model through a real world mobility trace for large geographical areas," in Proc. 8th ACM Int. Symp. MSWiM, Montreal, QC, Canada, Oct. 2005, pp. 174-177.

[17] W.-J. Hsu, K. Merchant, H.-W. Shu, C.-H. Hsu, and A. Helmy, "Weighted waypoint mobility model and its impact on ad hoc networks," ACM SIGMOBILE Mobile Comput. Commun. Rev., vol. 9, no. 1, pp. 59-63, Jan. 2005. 
[18] Q. Zheng, X. Hong, and J. Liu, "An agenda based mobility model," in Proc. 39th ANSS, Huntsville, AL, USA, Apr. 2006, pp. $188-195$.

[19] M. Musolesi and C. Mascolo, "A community based mobility model for ad hoc network research," in Proc. 2nd Int. Workshop REALMAN, Florence, Italy, May 2006, pp. 31-38.

[20] T. Litman. (2009). Safe travels: Evaluating mobility management traffic safety impacts. Transportation Research Board [Online]. Available: http://www.trb.org/Main/Blurbs/155263.aspx

[21] T. Litman, "Measuring transportation: Traffic, mobility, and accessibility," ITE J., vol. 73, no. 10, pp. 28-32, Oct. 2003.

[22] M. Behrisch, L. Bieker, J. Erdmann, and D. Krajzewicz, "Sumo-simulation of urban mobility: An overview," in Proc. 3rd Int. Conf. Adv. Syst. Simul., Barcelona, Spain, Oct. 2011, pp. 55-60.

[23] J. Yoon, B. D. Noble, M. Liu, and M. Kim, "Building realistic mobility models from coarse-grained traces," in Proc. 4th Int. Conf. MobiSys, Uppsala, Sweden, Jun. 2006, pp. 177-190.

[24] M. Kim, D. Kotz, and S. Kim, "Extracting a mobility model from real user traces," in Proc. 25th IEEE INFOCOM, Barcelona, Spain, Apr. 2006, pp. 1-13.

[25] N. Banerjee, M. Corner, D. Towsley, and B. Levine, "Relays, base stations, and meshes: Enhancing mobile networks with infrastructure," in Proc. 14th ACM MobiCom, San Francisco, CA, USA, Sep. 2008.

[26] F. Bai and B. Krishnamachari, "Spatio-temporal variations of vehicle traffic in VANETs: Facts and implications," in Proc. 6th ACM VANET, Beijing, China, Sep. 2009.

[27] H. Zhu et al., "Impact of traffic influxes: Revealing exponential inter-contact time in urban VANETs," IEEE Trans. Parallel Distrib. Syst., vol. 22, no. 8, pp. 1258-1266, Aug. 2011.

[28] K. Lee et al., "Max-contribution: On optimal resource allocation in delay tolerant networks," in Proc. 29th IEEE INFOCOM, San Diego, CA, USA, Mar. 2010, pp. 1-9.

[29] H. Zhu et al., "Recognizing exponential inter-contact time in VANETs," in Proc. 29th IEEE INFOCOM, San Diego, CA, USA, Mar. 2010, pp. 1-5.

[30] Y. Li, D. Jin, Z. Wang, L. Zeng, and S. Chen, "Exponential and power law distribution of contact duration in urban vehicular ad hoc networks," IEEE Sig. Process. Lett., vol. 20, no. 1, pp. 110-113, Jan. 2013.

[31] F. P. Kelly, "Networks of queues with customers of different types," J. Appl. Probab., vol. 12, no. 3, pp. 542-554, Sep. 1975.

[32] D. S. Menasche, A. A. A. Rocha, B. Li, D. Towsley, and A. Venkataramani, "Content availability and bundling in swarming systems," in Proc. 5th CoNEXT, Rome, Italy, Dec. 2009, pp. 121-132.

[33] D. Wu, Y. Liu, and K. W. Ross, "Queueing network models for multi-channel P2P live streaming systems," in Proc. 28th IEEE INFOCOM, Rio de Janeiro, Brazil, Apr. 2009, pp. 73-81.

[34] F. Ashtiani, J. A. Salehi, and M. R. Aref, "Mobility modeling and analytical solution for spatial traffic distribution in wireless multimedia networks," IEEE J. Sel. Areas Commun., vol. 21, no. 10, pp. 1699-1709, Dec. 2003.

[35] K. Kim and H. Choi, "A mobility model and performance analysis in wireless cellular network with general distribution and multicell model," Wireless Personal Commun., vol. 53, no. 2, pp. 179-198, 2010.

[36] M. Li, H. Zhu, Y. Zhu, and L. M. Ni, "ANTS: Efficient vehicle locating based on ant search in ShanghaiGrid," IEEE Trans. Veh. Technol., vol. 58, no. 8, pp. 4088-4097, Oct. 2009.

[37] K. Kise, A. Sato, and M. Iwata, "Segmentation of page images using the area voronoi diagram," Comput. Vis. Image Understand., vol. 70, no. 3, pp. 370-382, Jun. 1998.

[38] E. D. Lazowska, J. Zahorjan, G. S. Graham, and K. C. Sevcik, Quantitative System Performance: Computer System Analysis Using Queueing Network Models. Upper Saddle River, NJ, USA: PrenticeHall, 1984.
[39] J. G. Kemeny and J. L. Snell, Finite Markov Chains. New York, NY, USA: Springer, 1960.

[40] B. S. Everitt, The Cambridge Dictionary of Statistics, 3rd ed. Cambridge, U.K.: Cambridge University Press, 2006.

[41] G. Zhang, X. Wang, Y.-C. Liang, and J. Liu, "Fast and robust spectrum sensing via Kolmogorov-Smirnov test," IEEE Trans. Commun., vol. 58, no. 12, pp. 3410-3416, Dec. 2010.

[42] D. Câmara, N. Frangiadakis, F. Filali, and C. Bonnet, "Vehicular delay tolerant networks," in Handbook of Research on Mobility and Computing: Evolving Technologies and Ubiquitous Impacts, M. M. Cruz-Cunha and F. Moreira, Eds. Hershey, PA, USA: IGI Global, 2011, pp. 356-367.

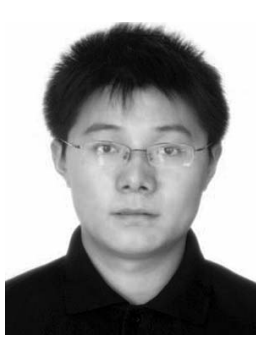

Yong Li (MŠ09) received the B.S. degree in electronics and information engineering from the Huazhong University of Science and Technology, Wuhan, China, in 2007, and the Ph.D. degree in electronic engineering from Tsinghua University, Beijing, China, in 2012. During July to August 2012 and 2013, he was a Visiting Research Associate with Telekom Innovation Laboratories and Hong Kong University of Science and Technology, respectively. During 2013 to 2014, he was a Visiting Scientist with the University of Miami, Coral Gables, FL, USA. He is currently a Faculty Member of the Department of Electronic Engineering, Tsinghua University. His research interests are in the areas of networking and communications, including mobile opportunistic networks, device-to-device communication, software-defined networks, network virtualization, and future Internet. He has served as a Technical Program Committee (TPC) Chair for the WWW workshop of Simplex 2013 and the TPC for several international workshops and conferences. He is a Guest Editor of the ACM/Springer Mobile Networks and Applications, Special Issue on Software-Defined and Virtualized Future Wireless Networks. $\mathrm{He}$ is currently an Associate Editor of the EURASIP Journal on Wireless Communications and Networking. He was a recipient of the Outstanding Postdoctoral Researcher, Outstanding Ph.D. Graduates, and Outstanding Doctoral Thesis awards from Tsinghua University. His research is granted by the Young Scientist Fund of the Natural Science Foundation of China, the Post-Doctoral Special Fund of China, and industry companies of Hitachi and ZET. He is a member of the IEEE.

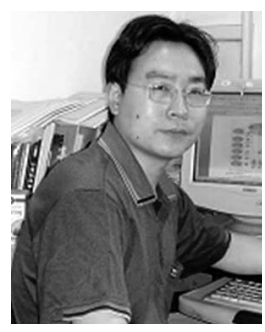

Depeng Jin received the B.S. and Ph.D. degrees from Tsinghua University, Beijing, China, in 1995 and 1999, respectively, both in electronics engineering. He is an Associate Professor at Tsinghua University and a Vice Chair of the Department of Electronic Engineering. He was awarded National Scientific and Technological Innovation Prize (Second Class) in 2002. His current research interests include telecommunications, high-speed networks, ASIC design, and future Internet architecture. He is a member of the IEEE. 


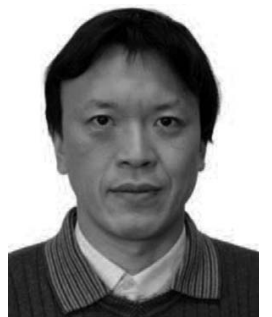

Zhaocheng Wang (M'09-SM-'11) received the B.S., M.S., and Ph.D. degrees from Tsinghua University, Beijing, China, in 1991, 1993, and 1996, respectively. From 1996 to 1997, he was with Nanyang Technological University (NTU), Singapore as a Post-Doctoral Fellow. From 1997 to 1999, he was with OKI Techno Centre Pte. Ltd., Singapore, firstly, as a Research Engineer and then as a Senior Engineer. From 1999 to 2009, he was with SONY Deutschland $\mathrm{GmbH}$, firstly as a Senior Engineer and then as a Principal Engineer. He is currently a Professor in the Department of Electronic Engineering, Tsinghua University. His current research interests include wireless communications, digital broadcasting, and millimeter wave communications. He received 22 granted U.S./E.U. patents and has published over 60 technical papers. He has served as Technical Program Committee co-chair/member of many international conferences. He is a senior member of the IEEE and a Fellow of IET.

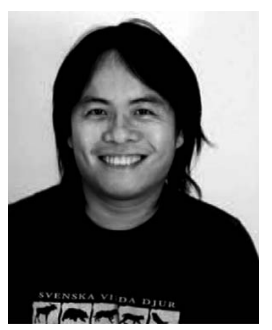

Pan Hui received the B.Eng. and M.Phil. degrees both from the Department of Electrical and Electronic Engineering, University of Hong Kong, Hong Kong, and the Ph.D degree from Computer Laboratory, University of Cambridge, Cambridge, U.K. He is currently a Faculty Member in the Department of Computer Science and Engineering, the Hong Kong University of Science and Technology, Hong Kong, where he directs the System and Media Lab. He also serves as a Distinguished Scientist of Telekom Innovation Laboratories (T-labs), Germany, and an Adjunct Professor of social computing and networking at Aalto University, Helsinki, Finland. Before returning to Hong Kong, he spent several years in T-labs and Intel Research Cambridge. He has published over 100 research papers and has several granted and pending European patents. He has founded and chaired several IEEE/ACM conferences/workshops, and served on the Technical Program Committee of numerous international conferences and workshops including IEEE Infocom, SECON, MASS, Globecom, WCNC, and ITC. He is a member of the IEEE.

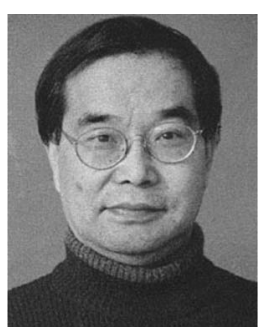

Lieguang Zeng received the B.S. degree from Tsinghua University, Beijing, China, in 1970. Since 1970, he has been with the Department of Electronic Engineering, Tsinghua University, Beijing, where he is currently a Professor. $\mathrm{He}$ has been awarded National Scientific and Technological Innovation Prize (Second Class), in 1987, 1991 and 2002. He has also been awarded provincial and ministerial level science and technology progress prize eight times. He has published over 150 research papers, and obtained four patents. His research interests include telecommunications, high-speed networks, ASIC design, and future internet architecture.

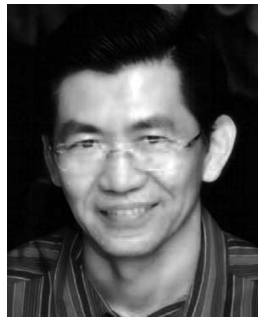

Sheng Chen (M'90-SM'97-F'08) received the B.Eng. degree from the East China Petroleum Institute, Dongying, China, in January 1982, and the Ph.D. degree from the City University, London, in September 1986, both in control engineering. In 2005, he was awarded the higher doctorate degree, Doctor of Sciences (D.Sc.), from the University of Southampton, Southampton, U.K. From 1986 to 1999, he held research and academic appointments at the Universities of Sheffield, Edinburgh and Portsmouth, all in the U.K. Since 1999, he has been with Electronics and Computer Science, the University of Southampton, where he currently holds the post of Professor at Intelligent Systems and Signal Processing. $\mathrm{He}$ is a Distinguished Adjunct Professor at King Abdulaziz University, Jeddah, Saudi Arabia. He is a Chartered Engineer (C.Eng.) and a Fellow of IET (FIET). His current research interests include adaptive signal processing, wireless communications, modelling and identification of nonlinear systems, neural network and machine learning, intelligent control system design, evolutionary computation methods, and optimisation. He has published over 470 research papers. He is an ISI highly cited researcher in the engineering category in March 2004. $\mathrm{He}$ is a fellow of the IEEE.

$\triangleright$ For more information on this or any other computing topic, please visit our Digital Library at www.computer.org/publications/dlib. 